\title{
Investigating physics faculty's reasoning about inequities in undergraduate physics education
}

\author{
Chandra Turpen, ${ }^{1}$ Angela Little, ${ }^{1,2}$ and Vashti Sawtelle ${ }^{2}$ \\ ${ }^{I}$ Department of Physics, University of Maryland, College Park, 082 Regents Dr., College Park, MD 20742, USA \\ ${ }^{2}$ Department of Physics \& Astronomy, Michigan State University, Lyman Briggs College, 567 Wilson Rd., East Lansing, MI \\ 48824, USA
}

\begin{abstract}
Grappling with inequities in physics learning is a central responsibility of physics instructors, yet little is known about how faculty do this important work. In a pilot interview study with twelve physics faculty, we asked faculty about: (a) their awareness of student experiences with bias, discrimination, and hardship and (b) the fairness of various common classroom instructional practices. Our analysis of three case studies shows faculty noticing inequities in their own classrooms and listening carefully to students' experiences with racialized, gendered, and/or socioeconomic injustices. We find that this listening and noticing supports faculty in: (1) taking actions to shift classroom dynamics, (2) reinterpreting themselves in relation to physics and critiquing physics culture, (3) linking up students with institutional resources, and (4) critiquing institutional practices. Therefore, we argue that faculty's listening and noticing not only supports faculty growth, but also has the potential to impact students, and the systems that students are embedded in.
\end{abstract}

\section{INTRODUCTION}

Preparing faculty to grapple with inequities in physics learning is important because it is a central responsibility of physics instructors [1] and faculty report needing training in these areas (e.g. in our pilot study 12/12 interviewees reported that they would welcome more training). Yet, there is little research on how physics faculty reason about diversity, equity and inclusion [2].

Historically, our PER community has taken a constructivist approach, aiming to build from what educators already know, in supporting the professional development (PD) of college faculty. To support such endeavors, researchers have investigated how physics faculty reason about instructional strategies [3,4], assessments [5], and problem-solving [6]. Following this tradition, we assume that physics faculty will have resources for thinking about equity and marginalization [7]. In order to design effective PD experiences for physics faculty, we need to know more about where faculty are coming from and the concrete equity dilemmas they face.

Researchers in higher education have documented that faculty and staff in collegiate institutions can bring a variety of cognitive frames to bear in making sense of racial inequities in educational outcomes: a diversity frame, a deficit frame, and an equity frame [8]. In the diversity frame, "individuals may embrace diversity [differences] but not take into account racial achievement patterns," and in the deficit frame individuals tend to "take note of racial achievement patterns but treat them as 'natural' in the light of the individuals' cultural, socioeconomic, and educational backgrounds" [pg. 102, Ref. 8]. Individuals that are reasoning with an equity frame "are more prone to notice and question patterns of educational outcomes, and they are also more likely to view inequalities in the context of a history of exclusion, discrimination, and educational apartheid" [pg. 102, Ref. 8].
From a situated resources perspective [9], we assumed that it would be possible to cue up any or all of these frames for physics instructors. Based on other preliminary work [10], we predicted that interview prompts that began with statistical patterns about attrition would likely cue up a pipeline metaphor of making sense of these data. For this reason, we structured our investigation around probing for faculty's awareness of student stories of bias, discrimination, and hardship, where we saw the most potential for producing reasoning that deviated from a pipeline metaphor and as a result open up space for drawing on equity frames.

Through our analysis of exemplary cases (where equity frames emerged), we will show evidence of faculty listening to students and noticing inequities. Using a progressive refinement of hypotheses analytic framework [11], we show how this listening and noticing supports faculty in: (1) taking actions to shift classroom dynamics, (2) reinterpreting themselves in relation to physics and critiquing physics culture, (3) linking up students with institutional supports, and (4) critiquing institutional practices. Through these pathways, we argue that faculty's listening and noticing not only supports faculty growth, but also has the potential to impact students, and the systems that students are embedded in (i.e. physics culture and institutional structures). This analysis illustrates how some instructors are doing the important work of grappling with inequities in physics learning.

\section{OUR STUDY AND METHODS}

We designed a pilot interview study with twelve physics faculty where we asked about: (a) their awareness of students' experiences with bias, discrimination, and hardship and (b) the fairness of various common classroom instructional practices. Our interview sample was drawn from the list of participants from a recent implementation of the New Physics and Astronomy Faculty Workshop 
(NFW). Historically, the NFW has dedicated one session to building faculty's awareness of under-representation issues in physics and one session to sharing about APS initiatives directed at impacting these issues. Following their attendance at NFW, all participants from a single cohort were recruited to participate in an interview about, "the equity and fairness of [your physics program's] structures and practices." Following this initial contact 16 people volunteered, and we were able to schedule interviews with $75 \%$ of them $(\mathrm{N}=12)$. The remaining NFW participants did not respond to our initial inquiry. Our interview sample has a higher fraction of faculty from minority serving institutions (MSI) (41\% versus 22\%) and a higher fraction of female faculty $(50 \%$ versus $34 \%)$ than NFW as a whole. It is worth noting that NFW recruits primarily young faculty, so our interview sample skews toward representing younger faculty compared to the national population of physics faculty.

In the design of our interview prompts, we aimed to assess faculty's access to student stories of bias, discrimination, or hardship (e.g. "Think of former students or mentees that you cared about and saw as having the potential to succeed in physics/astronomy. As a faculty member or graduate student, have you had such a student/mentee experience hardship based on some aspect of their identity?"). We predicted that starting our interview with these rich touchstone stories would elicit more nuanced reasoning about marginalization and oppression, then asking faculty to explain the factors associated with underrepresentation issues in physics more generally. Later parts of the interview elicited faculty's experiences reflecting on inequities in their classrooms (e.g. "As a course instructor, have you ever had any moments in your classroom where the space wasn't as inclusive as you'd wanted it to be?") or of common instructional practices (e.g. "In many instructional settings, it is fairly typical for an instructor to call on the student that has their hand up first. In what ways, do you see this practice as equitable or inequitable?"). A principle that guided our semi-structured interviewing was aiming to unpack people's understandings rather than challenging their interpretations of events.

The particular analysis presented here is focused on three of our twelve interview participants who explicitly described having experienced personal growth in their thinking about equity and marginalization (i.e. exemplary cases). This subset of instructors was rarely observed to draw on deficit thinking about students [8] as their explanations of inequities in physics learning. Instead, we find these faculty to be listening to students' experiences with racialized, gendered, and/or socioeconomic injustices and noticing inequities in their own classrooms. Based on this observation, our analysis focused on exploring, "What does access to student stories and noticing inequities in the classroom do?" Our analysis of these three interviews [11] allowed us to identify the following pathways for faculty: (1) Noticing as inspiring reflection and actions to shift inequitable classroom dynamics, (2) Noticing as inspiring reinterpretation of self and critique of physics culture, (3) Student stories inspiring faculty to link up students with institutional resources and (4) Student stories inspiring faculty to critique institutional practices. In this short paper, we exemplify these pathways through excerpts from one of the three instructors, "Brigid." Brigid is an early career physics faculty member at a MSI state university that values teaching in its tenure process. She identifies as a white woman. We member-checked our interpretations with Brigid. The other two instructors both displayed 3-4 of the pathways described above, and our analysis of dialogue with them informed our construction of our themes.

\section{DATA ANALYSIS}

\section{A. Noticing as inspiring reflection and actions to shift inequitable classroom dynamics}

In the following excerpt of interview data from Brigid, she is reflecting on class participation. Initially, we see Brigid acknowledging general differences in how girls and boys act: "I notice that with it, it's almost like the girls, just in general, the girls just have so much less confidence than the boys. ...It's like, they'd be more, "Oh, no. You go ahead. I don't have the answer. Just, confidence is a big issue. Then I think the underrepresented minorities are really like that, too." In this initial example, Brigid frames the participation differences as girls' confidence issues. But then she proceeds to describe her observations of classroom dynamics, and sees the cockiness and over-eagerness of some boys as the flip side of the same coin:

"I really notice in the class, because you want to be like, 'Okay, pals. We can all do this together.' But then you see cocky guys and you want to be like, 'You're not as smart as you think you are.' It's really hard to pitch that. Like, 'This is really easy and accessible, but not to you, because you need to realize you're not that...' You know what I mean? Trying to hit both of those is really tricky. Trying to have an atmosphere... and I even find it hard because you're trying to call and response, and it's the same cocky dudes falling out of their chairs trying to tell you the answer, and then it can be hard to balance that out. ... and I'm completely generalizing because there are guys who are not that confident. But, It's really hard to manage that like, in between."

She observes this interactional dynamic in her classroom, finds it problematic, and is actively trying to think about how to address it.

Brigid also describes one effort that her college is undertaking outside of the classroom context that bears on these issues:

"We had this other thing this semester where we've been trying to work on community 
building with the women in science. We had [a gathering]. So, I [believe] ...that if you put people together, they're not going to talk. If you put them together doing other things, then they will chat.... [The girls is the class], they've been part of this bigger women science thing and the [gatherings]. They get really excited about it. So I think that's how they have more confidence in the classroom then. So that's been really good."

In this example, we see Brigid working outside of class contexts to build community among the women science majors. She describes seeing them become cohesive, which has implications for their participation in class ("they have more confidence in the classroom then"). Here she describes actively contributing to the construction of this community space and then looking for the possible impacts of these spaces on her girls' participation in the introductory physics classroom.

\section{B. Noticing as inspiring reinterpretation of self and critique of physics culture}

In this segment, Brigid describes having some semesters where the classroom dynamics seem to go really well and other semesters that don't go so well. She shares the following example: "I had a heckler guy, and he actually really intimidated me. I didn't shut him down, and it spread. ...even to the point one day I wrote like, 'Newton's Third Law' in words. They were like, 'Is that an 'S' at the end of 'Newton,' or a '5'?' I was like... It's not his Twitter handle. It's just Newton's Third Law."

Brigid sees these moments as challenging to manage and recognizes that these moments cue up her "raging imposter syndrome":

"from my experience, I find it really hard to respond to them. I don't respond to aggression very well. I just get really like, 'Oh, you're probably right. Okay. Shit.' ... I always feel like a fraud. Do you know what I mean? I always feel like someone's going to find me out and realize that I'm impersonating a physicist and they should be arrested or something. Sometimes, when a student is particularly cocky and asking a question, I just feel like, 'Oh, they just seem - they know I don't know.' It's really hard. It's hard. Sometimes - it's weird, actually. In my role here, I feel more like a role model here, if that makes any sense? Here, I guess, maybe it is because it's more underrepresented minorities, but they're very like, 'Oh, you're the professor.' So, I feel like, 'Okay. I need to keep my shit together, because I want to set an example for these other women that you don't bow down to people being aggressive.' But I find it really hard."

Here we see Brigid reflecting on her own inner dialogue that kicks in during classroom moments where she feels aggressively challenged by young men in her class. She describes how challenging it is to deal with these feelings of inadequacy and fears of being revealed as a fraud. We see her recommitting herself to "keeping her shit together" in order to serve as a role model, wanting students to see women that "don't bow down to people being aggressive."

Brigid precedes to connect these experiences in the classroom with her own prior experiences as a graduate student in physics. Brigid sees these kinds of classroom dynamics as related broader cultural dynamics in physics:

"when I came to grad school and heard how people talk to each other, I nearly died. If people are so aggressive, and it's that male characteristic, ...I gave a talk on something to my research group. The girl in the group was like, "Oh, excuse me, I'm sure you explained it already, but," and she asked this super hardcore awesome question. Then there was the guy who was like, "I think this should be a minus." He was just super aggressive. At the end, the advisor was like, "Great contributions, [guy].

Good work." Because that's - he heard that as being the legit physics, because that's what it sounds like, right? It's like the men beating their chest...".

In this quotation we hear Brigid reflecting on the aggressive nature of conversations in graduate school. She compares how a woman in the research group might go about framing a question in a research seminar through hedging, and presuming that she missed something that was already explained, when her question was quite profound. However when a man in the group aggressively corrected "minus signs," the research mentor applauded him. She recognizes this as a problematic (and sexist) feature of physics culture.

Across these episodes we see Brigid noticing problematic classroom events and noticing her own inner dialogue in such moments. Then she coordinates these moments with making sense of her own personal identity in physics. The result is that she reinterprets prior events from graduate school, and critiques physics culture.

\section{Student stories inspiring faculty to link up students with institutional resources}

The third theme that we identified in our data was how faculty's access to student stories inspired faculty to connect students with institutional resources. Based on Brigid's experiences at a previous US institution, Brigid describes approaching a student to find out "Why'd you miss the quiz?" From this conversation, Brigid reports learning her student is being stalked on campus (e.g. "She was like, 'Oh, I was coming to school and my boyfriend, my ex-boyfriend who's in his [fancy car] and I had to go back home again."') Brigid proceeds to describe her response to the situation, "I am going to swoop in. I am going to sort this shit out and I'm going to contact the university..."

Brigid describes linking her student up with some contacts within the university. At the same time Brigid is 
disappointed in the kind of support this specific student received from the institution: "then nothing really happened for us" and "I don't feel like the administration really helped her. Because eventually, I saw her. She was like, 'Oh, everything's okay now because he finally graduated.' I was like, 'Well, that didn't seem like a great solution.", and "...So sometimes I feel frustrated." In this recounting we see Brigid reporting access to more holistic information about the student that allowed her to understand her students' disrupted participation in coursework. Brigid then worked to connect the student up with other resources on campus, but was frustrated by the institution's response (at her former institution).

\section{Student stories inspiring faculty to critique institutional practices}

The fourth theme that we identified in our data was how faculty's access to student stories inspired faculty to critique institutional practices and supports. In one story Brigid describes an experience with a student, "I had a girl in class, and her tooth just fell out in the middle of an exam, like it cracked, and she was in a lot of pain. So, we go to the health center. Then she was like, 'There's no dentist who takes my Medicare insurance in town.", Brigid proceeds to describe how she finds this to be unacceptable. She says, "Can you really bring students into a town and not have facilities... I just feel like because we're a [MSI], and we get kudos for being diverse, and that's great and everything. But then I feel like you have to have, it's the extra things that you might not think of, that they need to have a dentist to go to... Or if the surrounding area's super racist, then how are you going to protect people against that? More holistic thinking than just, 'Oh, you need a scholarship'..."

In this quotation, we see Brigid being critical of the institution's lack of advocacy on the behalf of students to make sure that students had reasonable access to dental and medical care that they would inevitably need while attending college. She says that the university must take a more holistic approach rather than just assuming scholarships will be enough to support students' success.

\section{CONCLUSIONS}

We have identified productive resources that some faculty bring to thinking about issues of equity. Though we do not know the prevalence of these resources, we found evidence of faculty listening to students and noticing inequities. Through the reasoning pathways we identified, we argue that faculty's listening and noticing not only supports faculty growth, but also has the potential to impact students, and the systems that students are embedded in (i.e. physics culture and institutional structures).

PD leaders might use our findings in multiple ways. Brigid knows of institutional resources available to her students, however, PD efforts could help more new faculty become aware of student support services that exist on their campuses and encourage faculty to integrate these into syllabi and routine faculty advising. Brigid has also made significant progress in recognizing concrete cases of inequities, however we suspect that she would benefit from opportunities to collaboratively analyze these equity dilemmas and consider possible actions. We suspect that collaborative analysis of such equity dilemmas would offer entry points for many faculty who may still be learning to recognize these injustices. Lastly, Brigid's reasoning suggests that PD leaders could work with faculty towards models of critical care [12], where the systems students are embedded in are critiqued and influenced. We anticipate building faculty's capacity for critical care necessitates recognition of systemic injustices. We recognize that there is not universal acceptance of this fact amongst physics faculty. We look forward to working with others to continue to build equity-focused PD for physics faculty.

\section{ACKNOWLEDGEMENTS}

This work is supported by funding from NSF DUE-1431681. We appreciate Alice Olmstead, Gina Quan, Ayush Gupta, and Stephen Secules for their formative feedback. We also thank the physics faculty who participated and actively supported this research. Without their cooperation this project would not have been possible.
[1] G. Ladson-Billings, Educ. Res. 35, 7 (2006).

[2] A. Johnson, A. Hodari, and M. Ong. Presented at AAPT Summer Meeting. Sacremento, CA (2016).

[3] M. Dancy, C. Henderson, and C. Turpen, Phys. Rev. Phys. Educ. Res. 12, 010110 (2016).

[4] C. Turpen, M. Dancy, and C. Henderson, Phys. Rev. Phys. Educ. Res. 12, 010116 (2016).

[5] A. Madsen, S. McKagan, M. S. Martinuk, and E. C. Sayre, Phys. Rev. Phys. Educ. Res. 12, 010115 (2016).

[6] E. Yerushalmi, C. Henderson, K. Heller, P. Heller, and V. Kuo, Phys. Rev. ST PER 3, 020109 (2007).
[7] K. Lowenstein, Rev. Educ. Res. 79, 1 (2009).

[8] E. M. Bensimon, New Dir. High. Educ. 131 (2005).

[9] D. Hammer, Am. J. Phys. 64, 1316 (1996); D. Hammer, Am. J. Phys. 68, S52 (2000).

[10] A. Pawley and J. Hoegh, ASEE Annual Conference \& Exposition Proceedings (2011).

[11] R. A. Engle, F. R. Conant, and J. G. Greeno. In R. Goldman, R. Pea, B. Barron, and S. J. Derry (Eds), Video research in the learning sciences (pp. 239-254).

[12] R. Rolon-Dow, Am. Educ. Res. J. 42, 1 (2005). 